\title{
Carcinoid Tumor Presenting as Acute Coronary Syndrome: A Case Report
}

\author{
Yasser M. Bawazir ${ }^{\mathrm{a}}$, Sara S. Baghlaf ${ }^{\mathrm{a}, \mathrm{b}}$, Abdulrhman A. Abulaban ${ }^{\mathrm{a}}$, \\ Imran Joher ${ }^{\mathrm{a}}$
}

\begin{abstract}
Pulmonary carcinoid tumors are neuroendocrine tumors that have an incidence of 1.2-2.1/100,000 among general population. Forty percent of patients with disseminated disease experience cardiac involvement, whither due to carcinoid heart disease or due to the vasospastic effect of secreted serotonin. Acute coronary syndrome is one of the rare entities that carinoid patients can present with. Few cases are reported in literature with similar presentation. Herein we are reporting a case of a 45-year-old Yamani male patient presented to the emergency department of our hospital with chest pain for 12 hours duration. He also reported a history of watery diarrhea for the last four months. He was diagnosed to have acute coronary syndrome. Further radiological investigations showed numerous enlarged mediastinal lymph nodes. Biopsy showed moderately differentiated neuroendocrine tumor consistent with atypical carcinoid.
\end{abstract}

Keywords: Carcinoid; Bronchial; Acute coronary syndrome; Neuroendocrine; Vasospasm

\section{Introduction}

Pulmonary carcinoid tumors, originating from Kulchitsky's cells of the bronchial mucosa layer, are neuroendocrine tumors that have an incidence of 1.2-2.1/100,000 among general population. And it represents about one to five percent of all lung tumors [1].

Serotonin that is produced by the tumor cells can induce coronary vasoconstriction in atherosclerotic coronary arteries, in addition to the other polypeptides secreted by the tumor that can augment the vasospastic response [2].

\footnotetext{
Manuscript accepted for publication August 5, 2013

${ }^{\mathrm{a}}$ Faculty of Medicine, King Abdulaziz University, Jeddah, Saudi Arabia

${ }^{\mathrm{b}}$ Corresponding author: Sara S. Baghlaf, Faculty of Medicine, King Abdulaziz University, P.O. Box 80215 Jeddah 21589, Saudi Arabia.

Email: dr.s.baghlaf@gmail.com
}

doi: http://dx.doi.org/10.4021/jmc1216w
Forty percent of patients with disseminated disease experience cardiac involvement, with the subsequent morbidity and mortality [3]. Herein, we report a case of bronchial carcinoid tumor presenting with acute coronary syndrome.

\section{Case Report}

A 45-year-old Yemeni male patient, previously smoker, presented to the emergency department with a sudden retrosternal chest pain for twelve hour prior to his presentation. The pain was radiating to the left shoulder, associated with grade II (NYHA) dysponea. He denied orthopnea, paroxysmal nocturnal dysponea, palpitation, lower limb swelling, syncope, hemoptysis and fever. Night sweats and weight loss were absent. He reported a history of watery diarrhea for the last four months associated with a colicky abdominal pain relieved after defecation, with no history of hot flushes or wheezy chest. He denied illegal drugs or alcohol ingestion. He was a smoker for 25 years (one pack/day) and quit six months ago. His past medical history was not significant for other ischemic heart disease risk factors.

The patient had unremarkable physical examination. His Electrocardiography (ECG) showed $\mathrm{T}$ wave inversion and ST depression in V2-V6 (Fig. 1), with a troponin of $0.13 \mathrm{ng} /$ $\mathrm{mL}$. Other laboratory investigations were unremarkable.

A chest $\mathrm{x}$-ray reviled widened mediastinum with irregular cardiac borders and opacity over the right lower lung lobe.

The patient was treated for ischemic heart diseases in the emergency room then shifted to the Coronary care unit (CCU) and percutanous transluminal coronary angiography (PTCA) was scheduled on the next day. Echocardiogram showed ejection fraction of $50 \%$ with marked concentric hypertrophy and grade II diastolic dysfunction. All cardiac valves were normal.

PTCA showed normal coronary arteries with hyperconractile left ventricle and hypertrophy of the papillary muscles. Based on these results the patient was shifted to the medical ward.

Upon further investigation, computed tomography of the chest showed a large heterogeneous soft tissue mass overlying both ventricles with numerous enlarged Medias- 


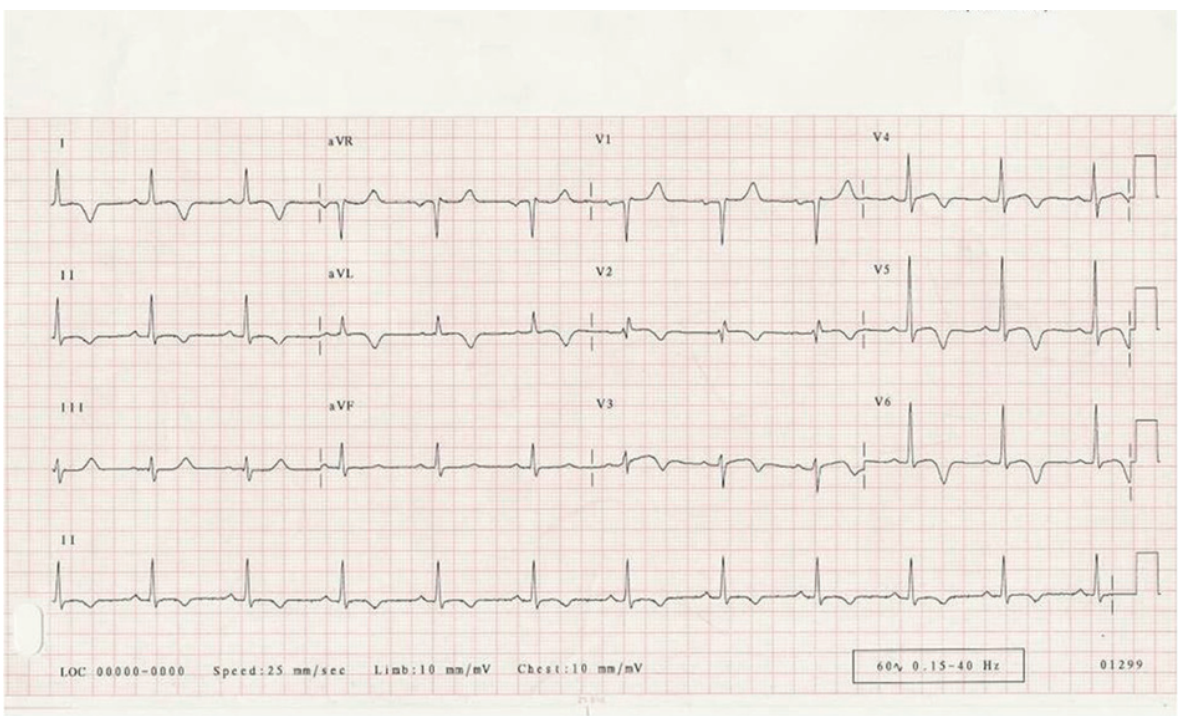

Figure 1. ECG changes of the patient; Initial ECG of the patient showing T wave inversion and ST depression in V2-V6.

tinal (Fig. 2), pre-tracheal, post-caval and azygoesophageal lymph nodes, in addition to a sub- centimeteric low density lesion in the left lobe of the liver.

Based on the previous results, a CT-guided mediastinal lymph node biopsy was taken and it showed moderately differentiated neuroendocrine tumor consistent with atypical carcinoid (Fig. 3), most likely metastasis from the lung.

Twenty four hour urinary collection of 5-hydroxyindoleacetic acid (5-HIAA) was elevated ( $24 \mathrm{mg} / \mathrm{dL})$.

The patient was started on Sandostatin $200 \mu \mathrm{g}$ subcutaneously three times daily for two weeks. He had dramatic improvement of his symptoms and then discharged home, to be followed in the clinic.

The patient remained asymptomatic at most recent follow up after discharge from the hospital.

\section{Discussion}

The presence of bronchial carcinoid producing serotonin and

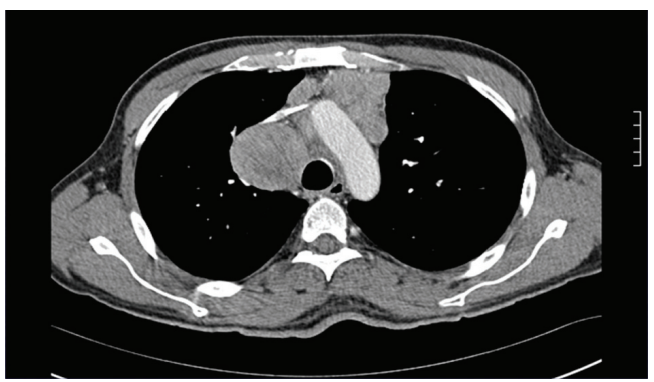

Figure 2. CT chest of the patient, showing enlarged Mediastinal lymph nodes. other vasoactive amines affect adversely on the heart, as the lung protective function of degrading 5-HIAA and reducing serotonin levels is lost. Which result in cardiac affection [4].

And the fact that the patient had no atherosclerotic risk factors other than smoking was a necessity for further workup.

In a study conducted by Bagheri et al, cough and hemoptysis were the most common symptoms of bronchial carcinoid; while our patient gave no previous history of such complains [5].

Biochemical diagnosis in our patient was attained by 24-hour urine sample collection for elevated 5-HIAA. It is an active metabolite of serotonin which is $100 \%$ specific for the diagnosis of carcinoid, but with a lows sensitivity $(73 \%)$ which can be explained by the fact that not all carcinoid tumors secrete serotonin [6].

In terms of management, Bagheri et al concluded their

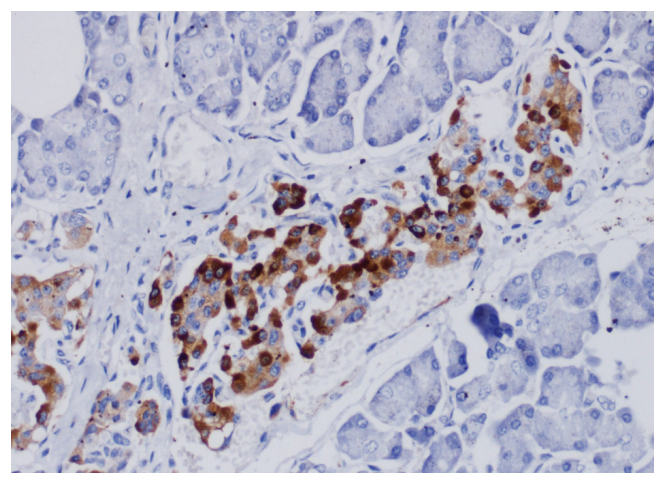

Figure 3. Mediastinal lymph node biopsy showing positive immune staining of chromogranin, indicating neuroendocrine tumor. 
study that surgical resection has been the standard treatment of non metastatic carcinoid tumors [5]. As our patient, hepatic metastasis is developed in $75 \%$ of carcinoid patients which requires multidisciplinary approach and the consideration of the available options such as surgical debulking, somatostatin analogue or systemic chemotherapeutic options [6]. And it has been shown that somatostatin analogue treatment can stabilize tumor growth in approximately $60 \%$ of patients over 11 months period [7].

Given the indolent course of carcinoid tumors, clinicians should be aware of the variable presentations that patients can manifest with. The lack of atherosclerotic risk factors and the presence of other symptoms suggestive of carcinoid syndrome, all can lead to further investigations and the diagnosis of carcinoid tumor. While surgical resection is the standard treatment in non metastatic diseases, multidisciplinary approach in required in metastatic carcinoid.

\section{Conflict of Interest}

The authors declare no conflict of interest.

\section{Source of Support}

The authors declare no source of support.

\section{References}

1. Koyyalamudi PL, Alfirevic A, Koch CG. An unusual presentation of carcinoid tumor. Anesth Analg. 2009;108(5):1463-1464.

2. Eapen DJ, Clements S, Jr., Block P, Sperling L. Metastatic carcinoid disease inducing coronary vasospasm. Tex Heart Inst J. 2012;39(1):76-78.

3. Evora PR, Bassetto S, Augusto VS, Vicente WV. Carcinoid heart valve disease: still a puzzle and a challenge. Arq Bras Cardiol. 2011;97(5):e111-112.

4. Marupakula V, Vinales KL, Najib MQ, Lanza LA, Lee HR, Chaliki HP. Occurrence of left-sided heart valve involvement before right-sided heart valve involvement in carcinoid heart disease. Eur $\mathrm{J}$ Echocardiogr. 2011;12(3):E18.

5. Bagheri R, Mashhadi M, Haghi SZ, Sadrizadh A, Rezaeetalab F. Tracheobronchopulmonary carcinoid tumors: analysis of 40 patients. Ann Thorac Cardiovasc Surg. 2011;17(1):7-12.

6. Pinchot SN, Holen K, Sippel RS, Chen H. Carcinoid tumors. Oncologist. 2008;13(12):1255-1269.

7. Aparicio T, Ducreux M, Baudin E, Sabourin JC, De Baere T, Mitry E, Schlumberger M, et al. Antitumour activity of somatostatin analogues in progressive metastatic neuroendocrine tumours. Eur J Cancer. 2001;37(8):10141019. 